Med Klin Intensivmed Notfmed 2018 • 113:53 https://doi.org/10.1007/s00063-017-0399-1 Online publiziert: 19. Dezember 2017 (c) Springer Medizin Verlag GmbH, ein Teil von Springer Nature 2017

\title{
Medizinische Klinik - Intensivmedizin und Notfallmedizin dankt den Gutachtern 2017
}

Die Schriftleitung dankt den Herausgebern der Zeitschrift und insbesondere allen weiteren Experten und Expertinnen für ihre Unterstützung im Jahr 2017 als wissenschaftliche Gutachter für Medizinische Klinik - Intensivmedizin und Notfallmedizin. Durch ihren Einsatz garantieren sie im Rahmen des Peer-Review-Verfahrens der eingereichten Manuskripte die hohe Qualität und klinische Relevanz der Zeitschrift.

Hervorgehoben seien Herr Dr. Matthias Winkler und Herr Prof. Dr. Guido Michels, die im Jahr 2017 die meisten Gutachten erstellt haben. Als Dankeschön erhalten sie ein Buch ihrer Wahl aus dem Programm des Springer-Verlages.

Dr. Maren Hermanns-Clausen, Freiburg

Carsten Hermes, Siegburg

Prof. Dr. Claus Peter Heußel,

Heidelberg

Prof. Dr. Hans Martin Hoffmeister, Solingen

Prof. Dr. Uwe Janssens, Eschweiler Univ.-Prof. Dr. Michael Joannidis, Innsbruck

Prof. Dr. Stefan John, Nürnberg

Prof. Dr. Achim Jörres, Köln

Arnold Kaltwasser, Reutlingen PD Dr. Clemens Kill, Marburg Prof. Dr. Stefan Kluge, Hamburg PD Dr. Alexander Koch, Aachen PD Dr. Matthias Kochanek, Köln Prof. Dr. Martin Köhrmann, Essen Prof. Dr. Roman Laszlo, Ulm

Dr. Henning Lemm, Siegen

Dr. Tobias Lindner, Berlin

Dr. Matthias Lubnow, Regensburg

Prof. Dr. Konstantin Mayer, Gießen Prof. Dr. Guido Michels, Köln Prof. Dr. Michael Oppert, Potsdam Prof. Dr. Sirak Petros, Leipzig Dr. Dr. Rudolf Pfab, München
PD Dr. Roland Prondzinsky, Merseburg Prof. Dr. Sebastian Reith, Aachen Prof. Dr. Reimer Riessen, Tübingen Dr. Martin Ruß, Dachau Prof. Dr. Bernd Saugel, Hamburg Prof. Dr. Dietmar Schneider, Leipzig Prof. Dr. Dr. h. c. Stefan Schwab, Erlangen Dr. Stefanie Siedler, Hamburg Kathrin Stöver, Leipzig Univ.-Prof. Dr. Thomas Staudinger, Wien

Prof. Dr. Helge Topka, München Dr. Hans-Joachim Trappe, Herne Prof. Dr. Arved Weimann, Leipzig Prof. Dr. Peter Weismüller, Hagen Prof. Dr. Tobias Welte, Hannover Dr. Tobias Wengenmayer, Freiburg Prof. Dr. Karl Werdan, Halle/Saale Dr. Michael Westhoff, Hemer Prof. Dr. Frank Willeke, Siegen Dr. Matthias Winkler, Merseburg Dr. Sebastian Wolfrum, Lübeck Prof. Dr. Christian Wrede, Berlin
Prof. Dr. med. Michael Haap, Tüb
Prof. Dr. Peter Heering, Solingen Ao Univ.-Prof. Dr. Gottfried Heinz, Wien 